\title{
GW23-e2342 THE RELATIONSHIP BETWEEN P2Y12 GENE POLYMORPHISMS AND CLOPIDOGREL RESISTANCE IN PATIENTS WITH CORONARY HEART DISEASE
}

doi:10.1136/heartjnl-2012-302920a.280

Zhang Wenbin, Chen Xiaoyan, Zhang Xinxia. Department of Cardiology, Shenzhen Futian Hospital Affiliated of Guangdong Medical College, Shenzhen

Objectives The antiplatelet efficacy of clopidogrel has a large interindividual variability. Polymorphisms in the P2Y12 gene have been suggested to contribute to this variability. The aim of this study was to investigate the contribution of P2Y12 genetic polymorphisms in rs6798347 in patients with coronary heart disease, and to explore the association between the polymorphism and clopidogrel resistance.

Methods A total of 91 patients with coronary artery disease were enrolled, which were diagnosed by coronary arteriography or coronary artery CT angiography. Platelet function was assessed by ADP-induced light-transmittance aggregometry after the patients received adequate clopidogrel pretreatment. Patients were divided into two groups (group of clopidogrel resistance and group of non-clopidogrel resistance) according to the ADP-induced platelet aggregation percentage. The genotype of P2Y12 (rs6798347) was detected by Mass ARRAY Time of Flight Mass Spectrometry. Compare the relationship of two groups of genotype and allele frequency distribution. And find the different in platelet aggregation percentage and severity of coronary artery lesion in different genotypes.

Results Based on the ADP-induced platelet aggregation percentage, 30 patients with clopidogrel resistance were selected. Remaining 61 were defined as non-clopidogrel resistance. Baseline characteristics were balanced between the two groups, except for the cholesterol total and low density lipoprotein $((4.11 \pm 1.12) \mathrm{mmol} / \mathrm{l}$ vs $(4.81 \pm 1.34) \mathrm{mmol} / \mathrm{l}$, $\mathrm{p}=0.015) ;((2.18 \pm 0.81) \mathrm{mmol} / \mathrm{l}$ vs $(2.57 \pm 0.89) \mathrm{mmol} / \mathrm{l}, \mathrm{p}=0.049)$. Ninety patients were genotyping successfully. In the clopidogrel resistance group, the polymorphisms of rs6798347 sites GG, AA, AG genotype frequencies are 14,5 and $10(48.28 \%, 17.24 \%$ and $34.48 \%)$; the other group are 33,5 and $23(54.10 \%, 8.20 \%$ and $37.70 \%)$. There is no different in genotype frequency between this two groups $(p>0.05)$. Also there is no different in ADP-induced platelet aggregation percentage and severity of coronary artery lesion in different genotypes ( $p>0.05)$.

Conclusions These date suggest that the Single Nucleotide Polymorphisms of P2Y12 in rs6798347 is not associated with clopidogrel resistance in patient with coronary heart disease. 